Article

\title{
A Neural Network-Based Interval Pattern Matcher
}

\section{Jing Lu ${ }^{1,2}$, Shengjun Xue ${ }^{1}$, Xiakun Zhang ${ }^{3,4, *}$ and Yang Han ${ }^{5,6}$}

1 School of Computer and Software, Nanjing University of Information Science and Technology, Nanjing 210044, China; E-Mails: lujing19810629@hotmail.com (J.L.); sjxue@nuist.edu.cn (S.X.)

2 Shanxi Meteorological Administration, Taiyuan 030006, China

3 National Meteorological Center of China Meteorological Administration, Beijing 100081, China

4 Environmental Modeling Center, NOAA/NWS/National Centers for Environmental Prediction, College Park, MD 20740, USA

5 School of Environmental Science and Engineering, Nanjing University of Information Science and Technology, Nanjing 210044, China; E-Mail: han_yang@outlook.com (Y.H.)

6 Earth System Science Interdisciplinary Center, University of Maryland, College Park, MD 20740, USA

* Author to whom correspondence should be addressed; E-Mail: zxk668@126.com; Tel./Fax: +86-25-5873-1184.

Academic Editor: Willy Susilo

Received: 7 May 2015 / Accepted: 14 July 2015 / Published: 17 July 2015

\begin{abstract}
One of the most important roles in the machine learning area is to classify, and neural networks are very important classifiers. However, traditional neural networks cannot identify intervals, let alone classify them. To improve their identification ability, we propose a neural network-based interval matcher in our paper. After summarizing the theoretical construction of the model, we take a simple and a practical weather forecasting experiment, which show that the recognizer accuracy reaches $100 \%$ and that is promising.
\end{abstract}

Keywords: neural network; interval; pattern matcher; weather forecasting

\section{Introduction}

Pattern recognition [1-3], which focuses on the recognition of patterns, is a branch of machine learning. In many cases, patterns are learned from training data, which is supervised learning, but when there is no training example provided, some pattern recognition algorithms can be used to discover previously unknown patterns, which is unsupervised learning [4-6]. 
Pattern recognition is to assign a class to a given input value or a set of values $[7,8]$. Generally speaking, pattern recognition algorithms aim to provide a reasonable answer for all possible inputs and to perform most likely matching of the inputs, taking into account their statistical variation. This is contrasted with pattern-matching algorithms, which look for exact output that matches to the input in a pre-existing pattern or patterns. A common example of a pattern-matching algorithm is regular expression matching [9-11]. In contrast to pattern recognition, pattern matching is generally not considered as a type of machine learning but a type of artificial intelligence, although pattern-matching algorithms can sometimes succeed in providing similar-quality output to the sort provided by pattern-recognition algorithms.

So far, there are some pattern-matching algorithms in the world, including ones for intervals. However, most of the interval-matching algorithms are used in much applied areas [12] and are highly related to specific features. In other words, these interval pattern matching methods are designed for specific problems, not for general ones.

The main contribution of the paper is to propose an interval pattern matcher that makes use of the neural network, and we call it a "neural network-based interval pattern matcher". This pattern matcher has the property that can identify a mathematical interval, instead of a real number. Experiments illustrate that neural networks can form the core of the "Neural Network-based Interval Pattern matcher" that can identify the patterns more quickly compared with the pattern-matching algorithm using a searching method once the training process finishes.

The organization of this paper is as follows. In Section 2, we briefly introduce the preliminaries of the paper: neural networks. Section 3 describes the modeling of a neural network-based interval pattern matcher. Section 4 is the experiment to test the classifier that we propose. Finally, conclusions are given.

\section{Preliminaries: Neural Networks}

Artificial neural networks (shown in Figure 1) are electronic neuron structures that simulate the neural structure of the brain. They process one training sample at one time and learn by comparing real output with targets. The errors from the initial training step are used to modify the network weights the second time around, and so on. Roughly speaking, an artificial neural network is composed of a set of input values $\left(x_{\mathrm{i}}\right)$, associated weights $\left(w_{i j}\right)$, and a function that sums the weights up and maps the results to the output vector $(y)$. Neurons are organized into layers. The input layer is composed of neurons that represent the feature variable vector $(x)$. The next layer is called the hidden layer; and there may be more than one hidden layer and more than one neuron in each hidden layer. The final layer is the output layer, where one node matches with one class for classification problems.

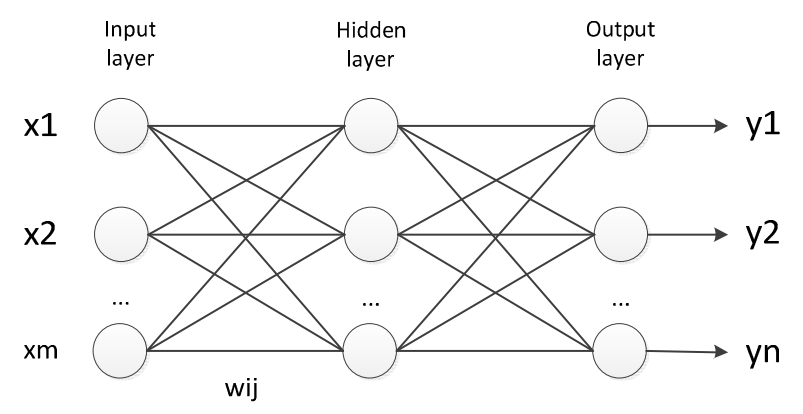

Figure 1. Artificial neural networks. 
Neural network is an important tool for classification $[13,14]$. Basically, a classifier takes objects as inputs and assigns each one to a class; objects are represented as vectors of features and classes are represented as class labels, such as 0 or 1 .

In the training phase, the correct class for each record is known, and the output nodes can therefore be assigned "class number" values - " 1 " for the node corresponding to the correct class, and " 0 " for the wrong class $[15,16]$. It is thus possible to compare the network's calculated output values with these "correct" (target) values, and calculate an error term for each node, which is the "Delta" rule. These errors are then used to adjust the weights in the hidden layers so that the output values will be closer to the "correct" (target) values for the next time. After the process of training, a single sweep forward through the network assigns a value to each output neuron, whose value is assigned to whichever class's node had the highest value.

It is well-known that there are several types of neural networks, each of which has unique functions. In our paper, we focus on the pattern matching ability using a neural network, and come up with a pattern matcher that is a neural network with additional data preparation layer. The traditional neural network is typical enough to be used to in our system illustrate our problem.

\section{Neural Network-Based Interval Classifier}

Rules [17-19] are often stated as linguistic IF-THEN constructions. It has the general form "IF $A$ THEN $B$ ", where $A$ is called the premise of the rule and B is the consequence. We use $(\mathrm{A} ; \mathrm{B})$ to denote this rule. Neural network can express such rule efficiently. However, it is more often seen that both $A$ and B take a more complex form.

Take the simple rule set below as an example

$$
\begin{aligned}
& \left(R_{1}\right) \text { IF } x_{1} \text { is Small AND } x_{2} \text { is Small, THEN } y \text { is Small; } \\
& \left(R_{2}\right) \text { IF } x_{1} \text { is Small' AND } x_{2} \text { is Small', THEN } y \text { is Small'. }
\end{aligned}
$$

There are two rules in this example, and the number of inputs is two. Small and Small' denote intervals over the universe of $x$ and $y . R_{1}$ can be denoted as (Small, Small; Small); $R_{2}$ can be denoted as (Small', Small'; Small').

Often, a continuous interval can be represented by some discrete examples. The discrete examples in the domain can be determined by interpolation. This idea reduces the processing complexity in our system using interval. For example, given the rule $R_{1}$, we need some specific value or (values) to depict the interval Small to minimize the complexity of it, neural network would face the difficulty that one input values would face more than one output value. Details are shown as the following:

If there is a one-to-many relationship between the inputs and targets in the training data, then it is not possible for any mapping to perform perfectly. One method is to calculate the average value for each interval. Any given network may or may not be able to approximate this mapping well at the beginning. However, it will form its best possible approximation to this mean output value when trained as well as possible.

One problem emerges when average features cannot distinguish one interval from the others. Sometimes, different intervals may have the same average values, especially for the intervals having overlap values. Whatever method we use, one interval should be distinguishable from the others on the 
limited domain related to the problem that we are trying to solve. For example, if our domain is $\{$ Large, Small, Middle \}, we need to distinguish these three intervals but do not need to distinguish others.

Here, we have to emphasize the concept of "relation". A "relation" is just a relationship among objects. If there are some persons in class and each person has their own height, the pair of one person and his/her name is a "relation". Besides, these pairs are "ordered", which means one comes first and the other comes second. The set of all the starting points is called "the domain" and the set of all the ending points is called "the range".

What should we notice is that all functions are relations but not all relations are functions. A function is a sub-classification of relation and a well-behaved relation, we mean that, given a starting point, we know exactly where to go; given an $x$, we get only and exactly one $y$. The relation $\{(7,8 ; 0),(7,8 ; 1)\}$ where "7" and " 8 " are input values and " 0 " and " 1 " are output values, is not a function since certain $x$ elements are paired with more than one unique $y$-element. However, the relation $\{(1 ; 2),(3 ; 2)\}$ where " 1 " and " 3 " are input values and " 2 " is an output value, is a function since certain $y$-elements value can be paired with more than one unique $x$-element.

It is well known that a neural network can approximate a function very well [20-22], but it cannot express the one-many mapping correctly since the weight changes would be compromised between different outputs given the same inputs. In a traditional neural network, the training error of a function would approximate zero when trained as well as possible [23-25]; while for the non-function relation, the error does not converge to a real number, but rather to an interval.

For example, if we are given a relation $\{(1 ; 2),(1 ; 3)\}$ and train the neural network using the training sample $(1 ; 2)$ and $(1 ; 3)$ alternately, at the beginning the weight change would simulate the relation $(1 ; 2)$ and then it would simulate the relation $(1 ; 3)$ and so forth. Thus, the weight changes would be a compromise among different outputs ("1" and "3") given the same inputs ("1") after a lot of iterations. When we input " 1 " to this well trained neural network, it would output a number between two and three randomly based on our research. Totally speaking, the range of the interval is closely related to the error interval.

Here, we make use of this error interval to decide if the training process is finished or not in the training process. Besides, we can also use this error interval and testing outputs to identify different interval output in the testing process. Specifically, the error interval would fall within an interval that is closely related to the error interval and output some values that fall within the interval itself. The network structure of this example is shown in Figure 2.

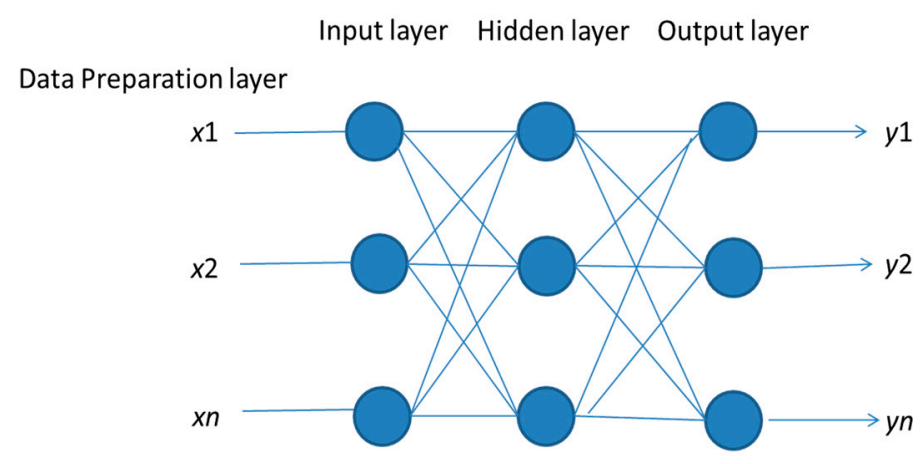

Figure 2. Neural network-based interval matcher. 


\section{Specification:}

Data preparation layer: This layer generates training samples from the raining rules. A suitable training sample is one which has values drawn from a distribution corresponding to a rule in the training rule base. (Training samples for rule $R_{1}$ must obey $R_{1}$.)

The main goal of the input layer is to generate some training samples randomly, each of which contains independent variable values $\left(x_{1}, \ldots, x_{n}\right)$ and values of the induced variable $(y)$ that obey the corresponding rule. We generate values for each of the variables by drawing from a rule distribution [26-28].

Using the example from Section 3, there are two independent variable values $\left(x_{1}, x_{2}\right)$ and one induced value $y$. To generate a training example for rule $R_{1}$, we would draw a sample for $x_{1}$ from the Small region of the domain of $X_{1} ; x_{2}$ would be drawn from the Small region for $X_{2}$, and $y$ would be drawn from the Small region of $Y$.

For example, we would like to approximate the relation $(A, B ; C)$, where $A$ is $\{1,2\}, B$ is $\{3,4\}$ and $C$ is $\{6,7\}$. We decompose this relation into $(1,3 ; 6),(1,3 ; 7),(1,4 ; 6),(1,4 ; 7),(2,3 ; 6),(2,3 ; 7),(2,4 ; 6),(2,4 ; 7)$.

Input layer: This layer is similar to standard neural networks with inputs from the data preparation layer.

Hidden layer: The function of this layer is similar to standard neural networks, except that we have separate networks for each set of input classes. The training itself is done using the Back-Propagation algorithm. During training, a training sample is only used to train a single network in the neural training layer, not all of them.

Output layer: This layer outputs an interval values. if we input $(1,3),(1,4),(2,3),(2,4)$, the output would be a number between 6 and 7 as would the others. Thus, we could estimate the interval $C$ based on the outputs of the corresponding well trained neural network.

\section{Experiments}

\subsection{Simple Test}

\subsubsection{Comparison Between Two Rules Using Our Program}

The reason why we conduct this experiment is to testify that the range of the interval is closely related to the error interval. We tested the range solution on the simple rules $R_{1}$ and $R_{2}$ :

Small is a set with the interval of $[0,10]$. In other words, it is part of the "Small". Table 1 depicts the experimental environment that we used for the tests.

Table 1. Experimental environment.

\begin{tabular}{ll}
\hline Algorithm to train the neural network & Back Propagation (BP) Algorithm \\
\hline Neural network structure & 4 layers having 3, 3, 3, and 1 neurons \\
\hline Experiment tool & Matlab \\
\hline
\end{tabular}

We first test out the range solution on $R_{1}$. For this example, the universal of all three variables is the interval $[0,100]$. To generate a test example, we simply generate a random three-tuple in $[0,100]^{3}$, and then check if this conforms to the stated rule $R_{1}$. (If it does not, we discard the training example.) 
In this experiment, we generate 42,803 training samples that satisfied the $R_{1}$ in the rule set. Using 30,000 training samples to train the neural network, we get the error values shown below in Figure 3 . Once the network finishes the training process, the error falls approximately within the interval [0-0.2].

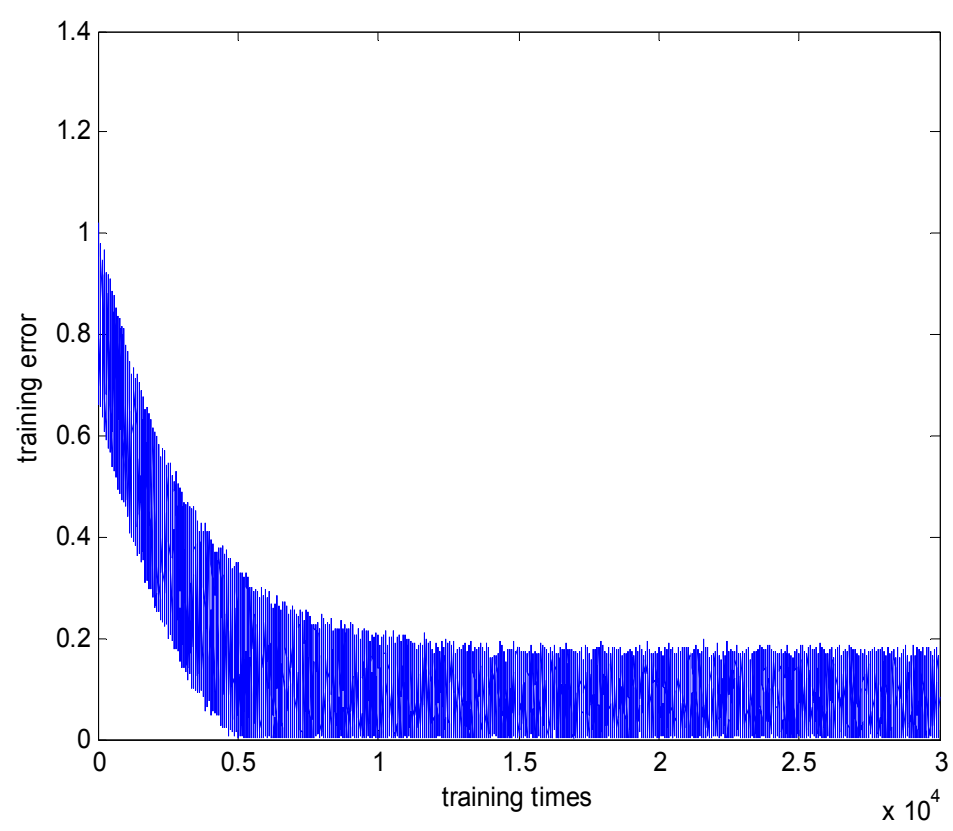

Figure 3. Training error using range solution of $R_{1}$.

Using the other 12,803 testing samples on the network, we get error values shown in Figure 4. We observe that the result approximately is between " 0 " to " 0.2 ". This means that the training process is successful and there is no over-fitting problem.

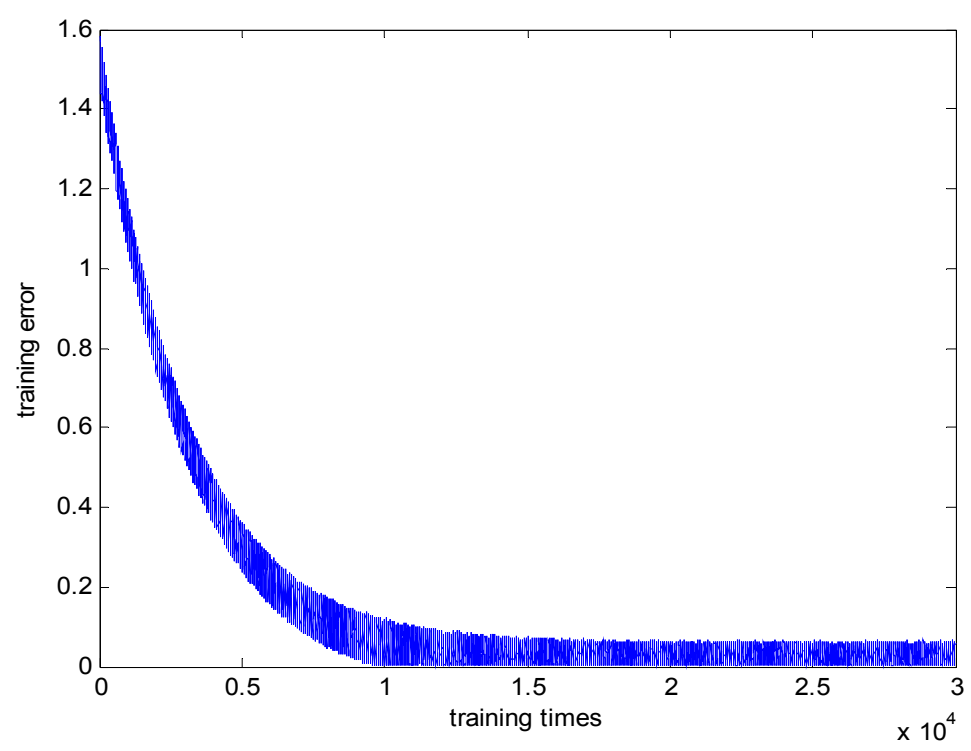

Figure 4. Training error using range solution of $R_{2}$.

We used another rule $R_{2}$ to test the range solution. Using 20,000 training samples to train, the training error falls approximately within an interval [0, 0.08], as shown in Figure 5. Using the other 10,000 testing samples to test, the testing error falls approximately within an interval [0,0.07], as shown in Figure 6. 


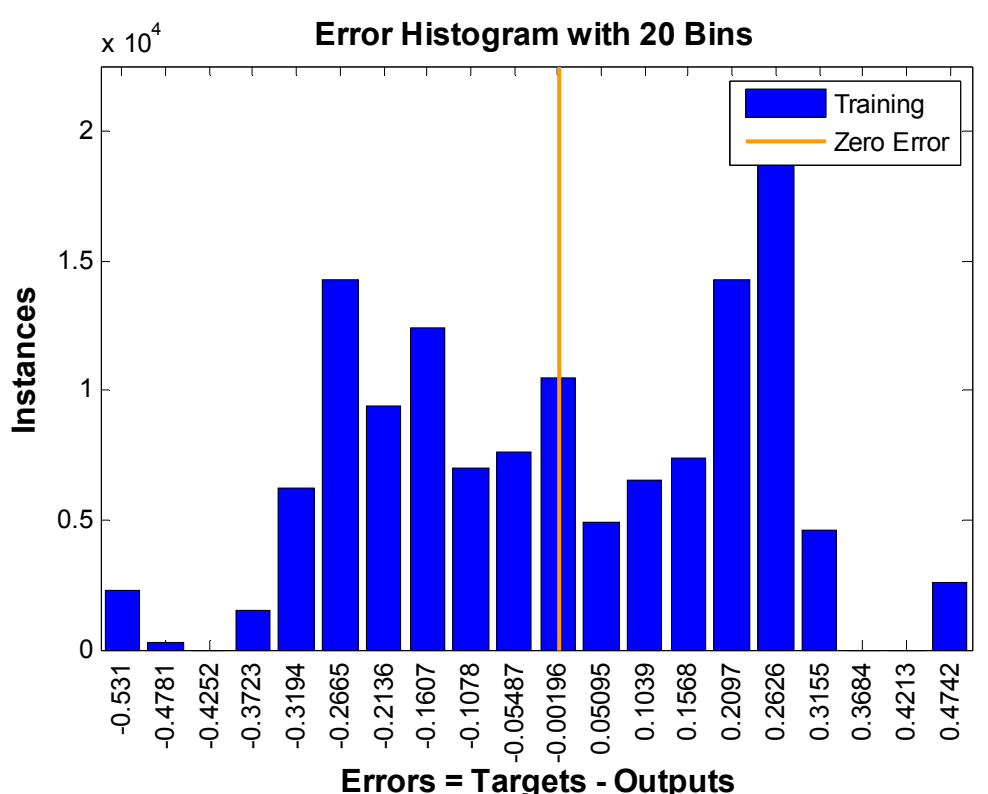

Figure 5. Training error using "traingdx" of $R_{1}$.

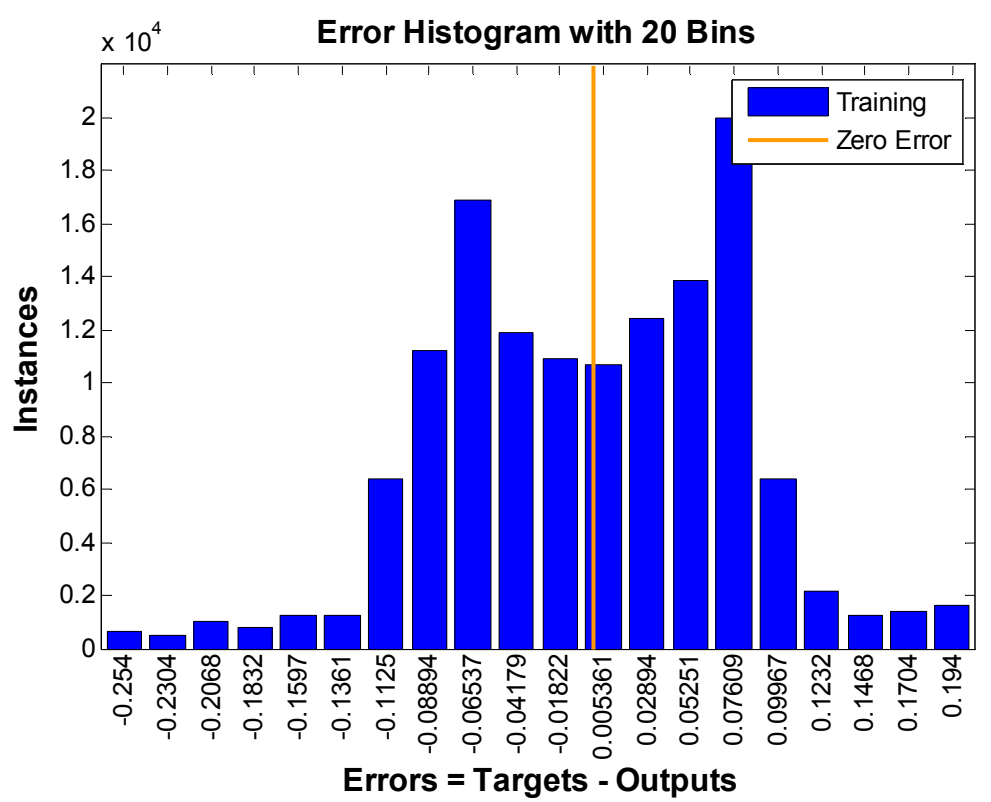

Figure 6. Training error using "traingdx" of $R_{2}$.

It is observed that both rules' training errors fall within an interval after getting a stable state, and the difference between the training errors of the two rules is the range of the error. The reason for this phenomenon is that the inputs of a training sample correspond to different output values. If the gap among the biggest output value and smallest output value is big, the range of the error is big; otherwise, the range of error is small.

Although the training error also falls within an interval for a function, the range of the interval will approach zero as long as the training times are sufficient. This is different from the non-function relation, whose training error range would not approach zero no matter how many training times the training process would take [28-30]. 


\subsubsection{Comparison Between Two Rules Using Matlab Toolbox}

Table 2 depicts the experimental environment that we used for the tests. We use "traingdx" to replace the general BP algorithm to test our results; use standard Matlab toolbox (version R2014a) to generate our results, instead of our own programming.

Table 2. Experimental environment.

\begin{tabular}{ll}
\hline Algorithm to train the neural network & $\begin{array}{l}\text { Gradient descent with momentum and adaptive learning rate backpropagation } \\
\text { algorithm (traingdx) }\end{array}$ \\
\hline Neural network structure & 4 layers having 3, 3, 3, and 1 neurons \\
\hline Experiment tool & Matlab neural network tool box \\
\hline
\end{tabular}

From Figures 5 and 6, we see the results are in accord with the last experimental results. Specifically, if we rotate the Figures 5 and 6 in clockwise direction about 90 degrees, these figures are almost the same as Figure 3 and Figure 4, respectively. With the increment of training times, the error would not decrease, but arrive to an interval, with the value vibrating within. The only difference is that Figures 5 and 6 have negative errors but Figures 3 and 4 do not. The reason for that is that we use absolute error to denote training errors.

\subsection{Practical Test}

From Shanxi Provincial Meteorological Bureau, China, we got the ground surface meteorological observation data in Taiyuan, a city of Shanxi Province, China from May 2007 to October 2007, May 2008 to October 2008 and May 2009 to October 2009, which are the rainy seasons in that city. The specific meteorological elements included the day's atmospheric pressure, relative humidity, dry and wet bulb temperature, wind speed and accumulated precipitation at 8 p.m. (Beijing Time, equivalent to 1200 UTC), which we took into our experiments.

Table 3. Classification of input conditions.

\begin{tabular}{|c|c|c|c|c|c|c|c|}
\hline \multicolumn{2}{|c|}{ Atmospheric pressure } & \multicolumn{2}{|c|}{$\begin{array}{c}\text { Dry and wet bulb } \\
\text { temperature }\end{array}$} & \multicolumn{2}{|c|}{ Relative humidity } & \multicolumn{2}{|c|}{ Wind speed } \\
\hline Rating & Value (hPa) & Rating & Value $\left({ }^{\circ} \mathrm{C}\right)$ & Rating & Value (\%) & Rating & Value (MPH) \\
\hline Moderate & $>940$ & Lowest & $<-10$ & Dry & {$[0,30)$} & Calm & $(0,2)$ \\
\hline Lower sliohtly & Г030 940$)$ & Lower & {$[-10,5)$} & Less dry & {$[30,50)$} & Light Air & {$[2,4)$} \\
\hline Lower si1gntiy & {$[930,940)$} & Moderate & {$[5,30)$} & & & Light Breeze & {$[4,7)$} \\
\hline Lower & {$[920,930)$} & Higher & {$[30,45)$} & Less humid & {$[50,70)$} & $\begin{array}{l}\text { Gentle } \\
\text { Breeze }\end{array}$ & {$[7,11)$} \\
\hline Lowest & $<920$ & Highest & $>45$ & Humid & {$[70,100]$} & $\begin{array}{c}\text { Moderate } \\
\text { Breeze }\end{array}$ & {$[11,17)$} \\
\hline
\end{tabular}

We took the day's atmospheric pressure, relative humidity, dry and wet bulb temperature, and wind speed observations at 8 p.m. (Beijing Time) as input conditions. Before the experiments, we classified the input meteorological conditions into different intervals according to their rating, which is shown in Table 3. Atmospheric pressure falls into four intervals shown in the first two columns of Table 3; relative 
humidity falls into four intervals shown in the third and fourth columns; dry and wet bulb temperature falls into five intervals shown in the fifth and sixth columns; wind speed falls into five intervals shown in the last two columns.

We also took the accumulated precipitation in the next 24 hours as outputs for the weather prediction models and used the precipitation observations to verify our results. Precipitation falls into five levels shown in Table 4.

Table 4. Classification of precipitation outputs.

\begin{tabular}{ll}
\hline Precipitation rating & Precipitation value (mm) \\
\hline Light rain & $(0,10.0)$ \\
Moderate rain & {$[10.0,24.9)$} \\
Heavy rain & {$[24.9,49.9)$} \\
Rainstorm & {$[49.9,99.9)$} \\
Heavy rainstorm & {$[99.9,249.0)$} \\
\hline
\end{tabular}

Experiments show that the accuracy of pattern matcher is $100 \%$, which is promising.

\section{Conclusions}

This paper presents an interval pattern matcher that can identify patterns with interval elements using neural networks. Our new system is based on a traditional neural network, but unlike previous neural networks, our system can handle interval inputs values and interval output values. This model is more convenient for some applications when compared with systems that require single real inputs and outputs.

We show that the new system is suitable for interval pattern matching, which look for exact output that matches to the input in pre-existing pattern or patterns. That is, our system can identify intervals patterns and are better than other pattern matchers using searching algorithms when trained as well as possible, since search-based matcher have to spend extra time in searching. In addition, our system can identify interval-based patterns while previous neural networks can only deal with simple patterns with simple real number inputs and outputs.

Finally, experiments show that the error intervals are highly closed with the output: the bigger the interval output range, the bigger the error range. In addition, practical experiments shows that the accuracy of identification is $100 \%$, which is promising.

As we know, the neural network can identify simple numbers, and we can now identify interval ones. This kind of matcher is important because most real world problems are better characterized by an interval rather than a single one.

\section{Acknowledgments}

This work was supported by the National Natural Science Foundation of China (No. 41275116, 41375121 and 41305079) and the Scientific Research and Innovation Plan for College Graduates of Jiangsu Province, China (No.CXZZ13_0500). 


\section{Author Contributions}

Jing Lu and Shengjun Xue conceived the study. Jing Lu conducted the experiments and analyzed the results. Jing Lu and Xiakun Zhang wrote the manuscript. Yang Han edited and revised the manuscript. All authors have read and approved the final manuscript.

\section{Conflicts of Interest}

The authors declare no conflict of interest.

\section{References and Notes}

1. Kawai, T.; Akira, S. The role of pattern-recognition receptors in innate immunity: Update on Toll-like receptors. Nat. Immunol. 2010, 11, 373-384.

2. Takeuchi, O.; Akira, S. Pattern recognition receptors and inflammation. Cell 2010, 140, 805-820.

3. Bunke, H.; Riesen, K. Recent advances in graph-based pattern recognition with applications in document analysis. Pattern Recognit. 2011, 44, 1057-1067.

4. Wright, J.; Yi, M.; Mairal, J.; Sapiro, G.; Huang, T.S.; Yan, S. Sparse Representation for Computer Vision and Pattern Recognition. Proceed. IEEE 2010, 98, 1031-1044.

5. Prest, A.; Schmid, C.; Ferrari, V. Weakly supervised learning of interactions between humans and objects. Pattern Anal. Mach. Intell. 2012, 34, 601-614.

6. Gureckis, T.M.; Love, B.C. Towards a unified account of supervised and unsupervised category learning. J. Exp. Theor. Artif. Intell. 2003, 15, 1-24.

7. Ulbricht, C.; Dorffner, G.; Lee, A. Neural networks for recognizing patterns in cardiotocograms Artif. Intell. Med. 1998, 12, 271-284.

8. Saba, T.; Rehman, A. Effects of artificially intelligent tools on pattern recognition. Int. J. Mach. Learn. Cybern. 2013, 4, 155-162.

9. Pedro, J.; García-Laencina, J.S.; Figueiras-Vidal, A.R. Pattern classification with missing data: A review. Neural Comput. Appl. 2010, 19, 263-282.

10. Santos, A.; Powell, J.A.; Hinks, J. Using pattern matching for the international benchmarking of production practices. Benchmarking: Int. J. 2001, 8, 35-47.

11. Ficara, D.; Di Pietro, A.; Giordano, S.; Procissi, G.; Vitucci, F.; Antichi, G. Differential encoding of DFAs for fast regular expression matching. IEEE/ACM Trans. Netw. 2011, 19, 683-694.

12. Huang, J.; Berger, T. Delay analysis of interval-searching contention resolution algorithms. IEEE Trans. Inf. Theoy. 1985, 31, 264-273.

13. Lin, C.; Chang, S. Efficient pattern matching algorithm for memory architecture. IEEE Trans. Very Larg. Scale Integr. Syst. 2011, 19, 33-41.

14. Yuan, H.; Wiele, C.F.V.D.; Khorram, S. An Automated Artificial Neural Network System for Land Use/Land Cover Classification from Landsat TM Imagery. Remote Sens. 2009, 1, 243-265; doi:10.3390/rs 1030243.

15. Levine, E.R.; Kimes, D.S.; Sigillito, V.G. Classifying soil structure using neural networks. Ecol. Model. 1996, 92, 101-108. 
16. Liu, H.; Chen, Y.; Peng, X.; Xie, J. A classification method of glass defect based on multiresolution and information fusion. Int. J. Adv. Manuf. Technol. 2011, 56, 1079-1090.

17. Chao, W.; Lai, H.; Shih, Y.I.; Chen, Y.; Lo Y.; Line, S.; Tsang, S.; Wu, R.; Jaw, F. Correction of inhomogeneous magnetic resonance images using multiscale retinex for segmentation accuracy improvement. Biomed. Signal Process. Control 2012, 7, 129-140.

18. Özbay, Y.; Ceylan, R.; Karlik, B. Integration of type-2 fuzzy clustering and wavelet transform in a neural network based ECG classifier. Expert Syst. Appl. 2011, 38, 1004-1010.

19. Orhan, U.; Hekim, M.; Ozer, M. EEG signals classification using the K-means clustering and a multilayer perceptron neural network model. Expert Syst. Appl. 2011, 38, 13475-13481.

20. Chen, L.; Liu, C.; Chiu, H. A neural network based approach for sentiment classification in the blogosphere. J. Informetr. 2011, 5, 313-322.

21. Mohamed, A.; Dahl, G.E.; Hinton, G. Acoustic modeling using deep belief networks. IEEE Trans. Audio Speech Lang. Process. 2012, 20, 14-22.

22. Feuring, T.; Lippe, W. The fuzzy neural network approximation lemma. Fuzzy Sets Syst. 1999, 102, 227-236.

23. Karlik, B.; Olgac, A.V. Performance analysis of various activation functions in generalized MLP architectures of neural networks. Int. J. Artif. Intell. Expert Syst. 2011, 1, 111-122.

24. Krizhevsky, A.; Sutskever, I.; Hinton, G.E. Imagenet classification with deep convolutional neural networks. Adv. Neural Inf. Process. Syst. 2012, 1, 1097-1105.

25. Hasiewicz, Z. Modular neural networks for non-linearity recovering by the Haar approximation. Neural Netw. 2000, 13, 1107-1133.

26. Lee, H.K.H. Consistency of posterior distributions for neural networks. Neural Netw. 2000, 13, 1107-1133.

27. Erhan, D.; Bengio, Y.; Courville, A. Why does unsupervised pre-training help deep learning? J. Mach. Learn. Res. 2010, 11, 625-660.

28. Shao, Y.; Lunetta, R.S. Comparison of support vector machine, neural network, and CART algorithms for the land-cover classification using limited training data points. ISPRS J. Photogramm. Remote Sens. 2012, 70, 78-87.

29. McCormack, L.; Dutkowski, P.; El-Badry A.M. Liver transplantation using fatty livers: Always feasible? J. Hepatol. 2011, 54, 1055-1062.

30. Cortes, M.; Pareja, E.; García-Cañaveras, J.C. Metabolomics discloses donor liver biomarkers associated with early allograft dysfunction. J. Hepatol. 2014, 61, 564-574.

31. Lloyd, C.; Grossman, A. The AIP (aryl hydrocarbon receptor-interacting protein) gene and its relation to the pathogenesis of pituitary adenomas. Endocrine 2014, 46, 387-396.

(C) 2015 by the authors; licensee MDPI, Basel, Switzerland. This article is an open access article distributed under the terms and conditions of the Creative Commons Attribution license (http://creativecommons.org/licenses/by/4.0/). 